\title{
THE ORDER OF CERTAIN CLASSES OF FUNCTIONS DEFINED IN THE UNIT DISK
}

\author{
D. C. RUNG
}

\section{Introduction ${ }^{1)}$}

Let $D$ denote the open unit disk in the complex plane and let $C$ be the boundary of $D$. If, for a given complex-valued function $f(z)$ defined in $D$, the existence of a subset $M$ of $C$ is known, with the linear measure of $M$ equal to $2 \pi$, as well as an estimate on the growth of $|f(z)|$ on sequences in $D$ which tends to a point of $M$, then such a result will be called a "statistical" result on order. This terminology is due to Lelong-Ferrand [3].

Such statistical-type results are known, for example, if the function $f(z)$ is the derivative of a univalent, holomorphic function (Seidel and Walsh [5], p. 141.) ; or if $f(z)$ is holomorphic in $D$ and omits two values there (Rung [4], p. 330). Both of these results depend upon first estimating the order of a holomorphic function $g(z)$ for which

$$
\iint_{n}|g(z)|^{2} d x d y<\infty .
$$

In sections 3,4 , and 5 of this paper we replace the function $|g(z)|$ in (1.0) by several arbitrary real-valued functions defined in $D$ and obtain statistical type results for these functions.

We conclude, in Section 6, by presenting examples of functions exhibiting this behavior.

\section{Terminology}

For $z_{0} \in D \cup C$ and $r>0$ set $D\left(z_{0}, r\right)=\left\{z \in D|| z-z_{0} \mid<r\right\}$. We proceed to introduce an outer measure on the plane. For $r \geq 0$ let $h(r)$ be a real valued, non-decreasing, continuous function with $h(0)=0, h(r)>0$ for $r>0$, and $h(\infty)>1$.

Received October 7, 1964.

1) Some results in this paper were part of the author's doctoral dissertation. The author would like to thank Professor W. Seidel for his advice during the preparation of this paper. The author was supported in part by a N.S.F. Grant G-9663, 
Definition 1. For a given set $E$ in the plane and fixed $\rho>0$ let $\left\{H\left(z_{j}, r_{j}\right)\right\}$ denote any denumerable family of open disks in the plane with center $z_{j}$ and radius $r_{j}, r_{j}<\rho$, which cover $E$. If $A_{\rho}$ is the inf $\left\{\sum_{j=1}^{\infty} h\left(r_{j}\right) \mid \cup_{j=1}^{\infty} H\left(z_{j}, r_{j}\right) \supset E, r_{j}<\rho\right\}$ define the $h$-measure of $E$ to be $h^{*}(E)=\lim _{\rho \rightarrow 0} A_{p}$.

Remark 1. In the case $h(r)=r^{k}, 0<k<2$, this defines on the plane the usual $k$-dimensional outer measure.

\section{Order of functions summable on $D$}

The following results depend upon a theorem of the author $[4, \mathrm{p} .324]$, which is closely related to a result of Lelong-Ferrand [3, pp. 20-23]. For completeness we state this theorem without proof.

Theorem A. Let $U(z)$ be a real-valued, non-negative, measurable function defined in D such that,

$$
\iint_{p} U(z) d x d y<\infty, \quad z=x+i y
$$

Then

$$
\lim _{r \rightarrow 0}\left[\frac{1}{h(r)} \iint_{D\left(e^{i \theta}, r\right)} U(z) d x d y\right]=0,
$$

except for at most a set of $e^{i \theta}$ of h-measure 0.

Remark 2. All integrals are to be considered as Lebesgue integrals.

In the following theorem an estimate is obtained on the order of such summable $U(z)$ on certain sequences in $D$.

Theorem 1. Let $U(z)$ satisfy the hypotheses of Theorem $A$. Then for every point of $C$, except possibly for a subset $S$ of $C$ of h-measure 0 , the following behavior occurs. Let $\left\{z_{n}\right\}$ be any sequence in $D$ tending to a point $e^{i \theta}$ of $C$ not in $S$. For any fixed $t, 0<t<1$, there exists a sequence of measurable sets $\left\{M_{n}(t)\right\}$ such that

i) $M_{n}(t) \subset D\left(z_{n},\left(1-\left|z_{n}\right|\right) t\right)$;

ii) $M_{n}(t)$ has positive two dimensional Lebesgue measure;

iii) if $\left\{\zeta_{n}\right\}$ is a sequence with

$$
\begin{aligned}
& \zeta_{n} \in M_{n}(t), n=1,2, \ldots, \\
& \lim _{n \rightarrow \infty} \frac{U\left(\zeta_{n}\right)\left(1-\left|\zeta_{n}\right|\right)^{2}}{h\left(\left|\zeta_{n}-e^{i \theta}\right|\right)}=0,
\end{aligned}
$$


Remark 3. It is obvious that $\zeta_{n} \in M_{n}(t)$ implies $\zeta_{n} \rightarrow e^{i \theta}$ as $n \rightarrow \infty$.

Remark 4. In this theorem, and in the sequel, we assume $h(r)$ also satisfies

$$
h(\alpha r) \leq K_{\alpha} h(r)
$$

$0 \leq r \leq \infty, \alpha>0$, and $K_{\alpha}$ is a positive constant depending only on $\alpha$. This property is not essential for this group of theorems and the necessary changes, if (3.0) is not assumed, will be obvious. For example, $h(r)=r^{k}$ satisfies this property.

Proof. Theorem A yields

$$
\lim _{r \rightarrow 0}\left|\frac{1}{h(r)} \iint_{D\left(e^{i \theta}, r\right)} U(z) d x d y\right|=0,
$$

except possibly for a subset of $C$ of $h$-measure 0 . Let $e^{i \theta}$ be a point at which (3.1) holds and suppose $\left\{z_{n}\right\}$ is any sequence in $D$ tending to $e^{i \theta}$. Further choose an arbitrary $t, 0<t<1$, which remains fixed during the course of the proof.

For $\zeta \in D\left(z_{n},\left(1-\left|z_{n}\right|\right) t\right)$, an easy calculation gives

$$
(1-t)\left|z_{n}-e^{i \theta}\right|<\left|\zeta-e^{i \theta}\right|<2\left|z_{n}-e^{i \theta}\right|,
$$

and

$$
(1-t)\left(1-\left|z_{n}\right|\right)<1-|\zeta|<(1+t)\left(1-\left|z_{n}\right|\right) .
$$

For the remainder of the proof set

$$
D\left(z_{n},\left(1-\left|z_{n}\right|\right) t\right) \equiv D_{n}, n=1,2, \ldots
$$

Since $U(z) \geq 0$, the right side of (3.2) gives

$$
\iint_{I_{n}} U(z) d x d y \leq \iint_{D\left(e^{i \theta}, 2 \mid z_{n}-e^{i \theta_{1}}\right)} U(z) d x d y .
$$

Theorem A, together with (3.0) and (3.4), enable us to conclude

$$
\left.\lim _{n \rightarrow \infty} \mid \frac{1}{h\left(\left|z_{n}-e^{i \theta}\right|\right)} \iint_{D_{n}} U(z) d x d y\right]=0 .
$$

The existence of the sets $M_{n}(t)$ is now demonstrated. Fix a positive integer $n$ and for this value of $n$ let $H$ denote the set of all points $\xi=u+i v$ contained in $D_{n}$ for which 


$$
U(\xi) \pi\left(1-\left|z_{n}\right|\right)^{2} t^{2}>\iint_{n_{n}} U(z) d x d y
$$

Since $U(z)$ is a measurable function $H$ is a measurable set. Further if the measure of $H$ were equal to $\pi\left(1-\left|z_{n}\right|\right)^{2} t^{2}$ integrating both sides of (3.6) over $H$ would give

$$
\iint_{H} U(\xi) d u d v>\iint_{D_{n}} U(z) d x d y=\iint_{H} U(z) d x d y
$$

which is impossible. Hence the measure of $H$ is less than $\pi\left(1-\left|z_{n}\right|\right)^{2} t^{2}$. Setting $M_{n}(t)$ equal to the complement of $H$ relative to $D_{n}$ we have, for $\zeta_{n} \in M_{n}(t)$, $n=1,2, \ldots$

$$
U\left(\zeta_{n}\right) \pi\left(1-\left|z_{n}\right|\right)^{2} t^{2} \leq \iint_{D_{n}} U(z) d x d y .
$$

We remark that the sequence of sets $\left\{M_{n}(t)\right\}$ depend upon the function $U(z)$, the sequence $\left\{z_{n}\right\}$, and the value $t$. In the sequel, if we introduce a function $U(z)$, a sequence $\left\{z_{n}\right\}$ and a value $t, 0<t<1,\left\{M_{n}(t)\right\}$ will always represent the above sequence of sets.

The proof is nearly complete since combining (3.5) and (3.7) gives

$$
\lim _{n \rightarrow \infty} \frac{U\left(\zeta_{n}\right)\left(1-\left|z_{n}\right|\right)^{2}}{h\left(\left|z_{n}-e^{i \theta}\right|\right)}=0 .
$$

However (3.8) may be revised to give

$$
\lim _{n \rightarrow \infty} \frac{U\left(\zeta_{n}\right)\left(1-\left|\zeta_{n}\right|\right)^{2}}{h\left(\left|\zeta_{n}-e^{i \theta}\right|\right)}=0
$$

if we refer to the right side of both (3.2) and (3.3) together with (3.0). Since this limit holds at every point $e^{i \theta}$ at which (3.1) is valid, the proof of Theorem 1 is complete.

If we restrict the sequence $\left\{z_{n}\right\}$ to approach $e^{i \theta}$ within some Stolz domain Theorem 1 can be reformulated. To this end let $S\left(e^{i \theta}, \alpha\right), 0<\alpha<\frac{\pi}{2}$. denote the symmetric Stolz domain at $e^{i \theta}$ of opening $2 \alpha$.

Corollary 1. Let $U(z)$ satisfy the hypotheses of Theorem 1 , and let $\left\{z_{n}\right\}$ be a sequence in $D$ tending to a point $e^{i \theta}$ but with $z_{n} \in S\left(e^{i \theta}, \alpha\right), n=1,2, \ldots$ for some $0<\alpha<\frac{\pi}{2}$. Then for any fixed $0<t<1$, and any sequence $\left\{\zeta_{n}\right\}, \zeta_{n} \in M_{n}(t)$, 
$n=1,2, \ldots$

$$
\lim _{n \rightarrow \infty} \frac{U\left(\zeta_{n}\right)\left(1-\left|\zeta_{n}\right|\right)^{2}}{h\left(1-\left|\zeta_{n}\right|\right)}=0
$$

except for at most a subset of $C$ of h-measure 0 .

Proof. An argument involving elementary geometry shows that, for any sufficiently small $\varepsilon>0$, there exists a positive integer $N_{0}$, depending on $\varepsilon$, such that for $n>N_{0}$

$$
D_{n} \subset S\left(e^{i \theta}, \alpha+\arcsin (t \cos \alpha)+\varepsilon\right) .
$$

This implies that for $n>N_{0}$ all $\zeta_{n}$ lie in the above Stolz domain, and, as is well known, then satisfy

$$
1 \leq \frac{\left|\zeta_{n}-e^{i \theta}\right|}{1-\left|\zeta_{n}\right|} \leq C
$$

for suitable constant $C$. Again referring to (3.0) as well as to the monotonicity of $h(r)$ we see that Theorem 1 can be restated to give Corollary 1 .

Remark 5. I am indebted to Professor W. Seidel for indicating (3.10).

Remark 6. Setting $h(r)=r$, which defines on $C$ the ordinary outer linear measure, the conclusion of Corollary 1 now reads

$$
\lim _{n \rightarrow \infty} U\left(\zeta_{n}\right)\left(1-\left|\zeta_{n}\right|\right)=0,
$$

and the exceptional subset of $C$ has linear measure 0 .

The question arises as to whether any estimate can be obtained for such summable $U(z)$ on the original sequence $\left\{z_{n}\right\}$. Several sufficient conditions are discussed in 4 and 5 .

\section{Sequentially subharmonic functions}

If we return to the proof of Theorem 1 we see that (3.7) relates the values of $U(z)$ at certain points in $D_{n}$ to the value of the integral of $U(z)$ over $D_{n}$. With this in mind we give

Definition 2. Let $U(z)$ be a real valued, non-negative measurable function defined in $D$. We say $U(z)$ is sequentially subharmonic in $D$ if, for each sequence $\left\{z_{n}\right\}$ in $D$ and for at least one value of $t, 0<t<1$, there exists a positive constant $K$ (which is a function of both the sequence and the value $t$ ) such that 


$$
U\left(z_{n}\right) \pi\left(1-\left|z_{n}\right|\right)^{2} t^{2} K \leq \iint_{n\left(z_{n},\left(1-\left|z_{n}\right|\right) t\right)} U(z) d x d y
$$

for $n=1,2, \ldots$

Remark 7. If $G(z)$ is a positive subharmonic function in $D$ then it is sequentially subharmonic in $D$ since $K$ can be chosen identically 1 for each sequence and each $0<t<1$.

The conclusion of Theorem 1 can be revised so that the sequence $\left\{\zeta_{n}\right\}$ is replaced by the original sequence $\left\{z_{n}\right\}$ if (3.7) is replaced by (4.0). This gives

Theorem 2. Let $U(z)$ be sequentially subharmonic in $D$ and suppose also

$$
\iint_{n} U(z) d x d y<\infty
$$

If $\left\{z_{n}\right\}$ is a sequence in $D$ which tends to a point $e^{i \theta}$ we have

$$
\lim _{n \rightarrow \infty} \frac{U\left(z_{n}\right)\left(1-\left|z_{n}\right|\right)^{2}}{h\left(\left|z_{n}-e^{i \theta}\right|\right)}=0
$$

except possibly for a set of $e^{i \theta}$ of h-measure 0 .

Corollary 2. Let the hypotheses of Theorem 2 be satisfied and in addition suppose the sequence $\left\{z_{n}\right\}$ approaches $e^{i \theta}$ within some Stolz domain at $e^{i \theta}$. Then

$$
\lim _{n \rightarrow \infty} \frac{U\left(z_{n}\right)\left(1-\left|z_{n}\right|\right)^{2}}{h\left(1-\left|z_{n}\right|\right)}=0
$$

except possibly for a set of $e^{i \theta}$ of h-measure 0 .

Remark 8. If $V(z)$ is a positive subharmonic function in $D$ then Theorem 2 applies to the function $U(z)=V^{p}(z), p \geq 1$, since $V^{p}(z)$ is also subharmonic. In the case $0<p<1, V^{p}(z)$ is still subharmonic provided $\log V(z)$ is. This generalizes a result of Gehring $[1, \mathrm{p} .77]$.

\section{Complex-valued functions summable over $D$}

Let $\phi(z)$ denote a complex-valued function defined in $D$. For any two points $a, b$ of $D$ set $\rho(a, b)$ equal to the non-euclidean (hyperbolic) distance between $a$ and $b$, i.e. $\rho(a, b)=1 / 2 \log \frac{|1-a \bar{b}|+|a-b|}{|1-a \bar{b}|-|a-b|}$.

Definition 3. Let $\left\{z_{n}\right\}$ be a sequence in $D$ which tends to a point of $C . \quad A$ complex-valued function $\phi(z)$ defined in $D$ is said to be close along $\left\{z_{n}\right\}$ if there 
exists a pair of positive real numbers $(\delta, M)$ such that if $\rho\left(z, z_{n}\right)<\delta$ then $\left|\phi(z)-\phi\left(z_{n}\right)\right|<M$, for each $n=1,2, \ldots$ If $\phi(z)$ is close along $\left\{z_{n}\right\}$ for all $\left\{z_{n}\right\}$ such that $\lim _{n \rightarrow \infty} z_{n}=e^{i \theta}, e^{i \theta} \in C$ (respectively $e^{i \theta} \in B, B$ a subset of $C$ with $h^{*}(B)=$ $\left.h^{*}(C)\right)$ then we say $\phi(z)$ is close along all sequences (respectively close along almost all sequences in the h-measure). When $h(r)=r$ we omit the phrase "in the $h$ measure."

For $z_{0} \in D$ and $\delta>0$ set $N\left(z_{0}, \delta\right)=\left\{z \mid \rho\left(z_{0}, z\right)<\delta\right\}$. This set of points is known to be an open Euclidean disk. Thus let $z^{\prime}$ denote the center and $\left(1-\left|z^{\prime}\right|\right) t^{\prime}$ the radius (both in the Euclidean geometry) of $N(z, \delta)$. We now indicate a connection between the non-Euclidean radius $\delta$ and the value $t^{\prime}$.

Lemma 1. Given the non-Euclidean disk $N(z, \delta)$ and its corresponding Euclidean representation $D\left(z^{\prime},\left(1-\left|z^{\prime}\right|\right) t^{\prime}\right)$ then

$$
t^{\prime}=\frac{K(1+|z|)}{1+K^{2}|z|}
$$

Hence as $|z| \rightarrow 1, t^{\prime} \rightarrow \frac{2 K}{1+K^{2}} \cdot K=\frac{e^{2 \delta}-1}{e^{2 \delta}+1}$.

Proof. If $z=r e^{i \theta}$, then the point $z_{2}$ on the boundary of $N(z, \delta)$ closest to $z$ in the Euclidean sense, is of the form $z_{1}=r_{1} e^{i \theta}, r_{1}>r$; the point $z_{2}$ furthest from $z$ is $z_{2}=r_{2} e^{i \theta}, r_{2}<r$. The point $z^{\prime}$ is also on the radius to $e^{i \theta}$ thus $z^{\prime}=r^{\prime} e^{i \theta}$, $r_{2}<r^{\prime}<r<r_{1}$. If we put $\left|z_{1}-z\right|=(1-|z|) t_{i}$ and $\left|z-z_{2}\right|=(1-|z|) t_{2}$, an elementary calculation gives $t_{1}=\frac{K(1+|z|)}{1+K|z|}$, and $t_{2}=\frac{K(1+|z|)}{1-K|z|}$. Thus the Euclidean radius of $N(z, \delta)$ is $\frac{\left|z_{1}-z_{2}\right|}{2}=(1-|z|)\left(\frac{t_{1}+t_{2}}{2}\right)$, and the Euclidean center $z^{\prime}=\frac{z_{2}+z_{1}}{2}$. Finally to find $t^{\prime}$ note that $\left(1-\left|z^{\prime}\right|\right) t^{\prime}=(1-|z|) \frac{\left(t_{1}+t_{2}\right)}{2}$ and a straightforward calculation gives the value $t^{\prime}$ in the Lemma.

This enables us to view a sequence of non-Euclidean disks $N\left(z_{n}, \delta\right)$, with $\lim _{n \rightarrow \infty}\left|z_{n}\right|=1$, as a sequence of Euclidean disks $D\left(z_{n}^{\prime}\left(1-\left|z_{n}^{\prime}\right|\right) t_{n}^{\prime}\right)$ where for $n>N_{0}, 0<\underline{t} \leq t_{n}^{\prime} \leq \bar{t}<1$, with $N_{0}, \underline{t}$ and $\bar{t}$ determined by Lemma 1 .

THEOREM 3. Let $\phi(z)$ be a complex-valued measurable function defined in $D$ which is close along almost all sequences in the h-measure and also, with $z=x+i y$,

$$
\iint_{n}|\phi(z)| d x d y<\infty
$$


Then, if $z_{n} \rightarrow e^{i \theta}$ as $n \rightarrow \infty$,

$$
\lim _{n \rightarrow \infty} \frac{\left|\phi\left(z_{n}\right)\right|\left(1-\left|z_{n}\right|\right)^{2}}{h\left(\left|z_{n}-e^{i \theta}\right|\right)}=0,
$$

except for at most a set of $e^{i \theta}$ of $h$-measure 0 .

Proof. Set $U(z)=|\phi(z)|$ and let $S$ denote the exceptional set of Theorem 1 for this $U(z)$. If $B$ is the set of all $e^{i \theta}$ relative to which $\phi(z)$ is close along sequences, $h^{*}(B)=h^{*}(C)$; hence setting $S_{1}=C-B, h^{*}\left(S_{1}\right)=0$, and $h^{*}\left(S_{2}\right)=0$ where $S_{2}=S \cup S_{1}$.

Let $\left\{z_{n}\right\}$ be any sequence in $D$ tending to a point $e^{i \theta}$ not in $S_{2}$. Since $\phi(z)$. is close along $\left\{z_{n}\right\}$ there exists a pair of positive real numbers $(\delta, M)$ such that $\rho\left(z, z_{n}\right)<\delta$ implies $\left|\phi(z)-\phi\left(z_{n}\right)\right|<M, n=1,2, \ldots$

Referring to Lemma 1 we consider the sequence $\left\{N\left(z_{n}, \delta\right)\right\}$ as a sequence of Euclidean disks $\left\{D\left(z_{n}^{\prime},\left(1-\left|z_{n}^{\prime}\right|\right) t_{n}^{\prime}\right)\right\}$. Since $t_{n}>\underline{t}, n>N_{0}$, we apply Theorem 1 with $t=\underline{t}$.

Thus for any sequence $\left\{\zeta_{n}\right\}, \zeta_{n} \in M_{n}(t), n=1,2, \ldots$,

$$
\lim _{n \rightarrow \infty} \frac{\left|\phi\left(\zeta_{n}\right)\right|\left(1-\left|\zeta_{n}\right|\right)^{2}}{h\left(\left|\zeta_{n}-e^{i \theta}\right|\right)}=0 .
$$

Since $M_{n}(t) \subset D\left(z_{n}^{\prime},\left(1-\left|z_{n}^{\prime}\right|\right) t^{\prime}\right), n=1,2, \ldots$, application of Lemma 1 gives $M_{n}(t) \subset N\left(z_{n}, \delta\right), n>N_{0}$. Since $\phi(z)$ is close along $\left\{z_{n}\right\}$.

$$
\left|\phi\left(z_{n}\right)\right| \leq M+\left|\phi\left(\zeta_{n}\right)\right|, n>N_{0}
$$

Combining (3.0), (3.2), (3.3) and (5.1)

$$
0 \leq \frac{\left|\phi\left(z_{n}\right)\right|\left(1-\left|z_{n}\right|\right)^{2}}{h\left(\left|z_{n}-e^{i \theta}\right|\right)} \leq \frac{M\left(1-\left|z_{n}\right|\right)^{2}}{h\left(\left|z_{n}-e^{i \theta}\right|\right)}+\frac{\left|\phi\left(\zeta_{n}\right)\right|\left(1-\left|\zeta_{n}\right|\right)^{2}}{h\left(\left|\zeta_{n}-e^{i \theta}\right|\right)}
$$

Now under the assumption that the $h$-measure of $C$ is positive (otherwise the statement of the theorem is vacuous)

$$
\lim _{z \rightarrow e^{i \theta}} \frac{(1-|z|)^{2}}{h\left(\left|z-e^{i \theta}\right|\right)}=0
$$

for all $e^{i \theta} \in C$. This follows by setting $U(z) \equiv 1$ in Theorem 2 and observing that if (5.3) holds for some $e^{i \theta}$, it holds for all $e^{i \theta}$.

The proof of Theorem 3 is completed by combining (5.0) and (5.3) with 
(5.2).

As Corollary 1 follows from Theorem 1 so also does the following Corollary follow from Theorem 3 .

Corollary 3. Under the hypotheses of Theorem 3, and supposing also that $\left\{z_{n}\right\}$ tends to $e^{i \theta}$ within some Stolz domain at $e^{i \theta}$,

$$
\lim _{n \rightarrow \infty} \frac{\left|\phi\left(z_{n}\right)\right|\left(1-\left|z_{n}\right|\right)^{2}}{h\left(1-\left|z_{n}\right|\right)}=0
$$

except possibly for a set of $e^{i \theta}$ of $h$-measure 0 .

\section{Examples to Theorem 1}

The following examples to Theorem 1 are constructed with $h(r)=r$.

ExAmple 1. Given an arbitrary countable subset $P$ of $C$ there exists a function $U_{1}(z)$ satisfying the hypotheses of Theorem 1 for which the exceptional set $S$ (of Theorem 1) contains $P$.

Let $\left\{e^{i p_{j}}\right\}$ be some enumeration of the points of $P$. For each point $e^{i p_{j}} \in P$ we will consider a sequence $\left\{z_{n}^{(j)}\right\}$ tending radially to $e^{i p_{j}}$; a sequence of disks $D\left(z_{n}^{(j)},\left(1-\left|z_{n}^{(j)}\right|\right) t_{j}\right)$; and a function $U_{1}(z)$ which takes the value $\frac{1}{(1-|z|)^{4 / 3}}$ in each disk and which is summable over $D$. If $\zeta_{n}^{(j)} \in D\left(z_{n}^{(j)},\left(1-\left|z_{n}^{(j)}\right|\right) t_{j}\right), n=1$, $2, \ldots$

$$
\lim _{n \rightarrow \infty} U_{1}\left(\zeta_{n}^{(j)}\right)\left(1-\left|\zeta_{n}^{(j)}\right|\right)=\infty
$$

Referring to Corollary 1 of Theorem 1 we see that the sets $M_{n}\left(t_{j}\right)$ do not exist for the sequece $\left\{z_{n}^{(j)}\right\}$; and $P$ is therefore a subset of the exceptional set $S$. We proceed to the details.

For a fixed $e^{i p_{j}} \in P$ consider a Stolz domain $S\left(e^{i p_{j}}, \alpha_{j}\right)$ of opening $2 \alpha_{j}$, $0<\alpha_{j}<\frac{\pi}{2}$, where $\left\{\alpha_{j}\right\}$ is any decreasing sequence of positive numbers satisfying

$$
\sum_{j=1}^{\infty} \alpha_{j}<\infty
$$

Let $\left\{z_{n}^{(j)}\right\}$ be a sequence approaching $e^{i p_{j}}$ radially with $\left|z_{n}^{(j)}\right|=1-\frac{1}{n^{2}}, n=1$, $2, \ldots$ If $t_{j}=\sin \alpha_{j}$ the disks $D\left(z_{n}^{(j)},\left(1-\left|z_{n}^{(j)}\right|\right) t_{j}\right), n=1,2, \ldots$, are easily seen to lie inside $S\left(e^{i p_{j}}, \alpha_{j}\right)$ (see figure 1 ). Hence-forth we use $D_{n}^{(j)}$ to denote these disks. 
New define

$$
U_{1}(z)=\left\{\begin{array}{l}
\frac{1}{(1-\mid z !)^{4 / 3}}, \quad z \in \bigcup_{j=1}^{\infty} \bigcup_{n=1}^{\infty} D_{n}^{(j)} ; \\
0, z \in D-\bigcup_{j=1}^{\infty} \bigcup_{n=1}^{\infty} D_{n}^{(j)} .
\end{array}\right.
$$

In order to compute the double integral of $U(z)$ over $D$ some estimates of the integral of $U(z)$ over each $D_{n}^{(j)}$ are required.

Let $\theta_{n}^{(j)}$ be that positive angle formed by the radius to $e^{i \rho_{j}}$ and the line segment from the origin tangent to the circumference of $D_{n}^{(j)}$ (See figure 1.).

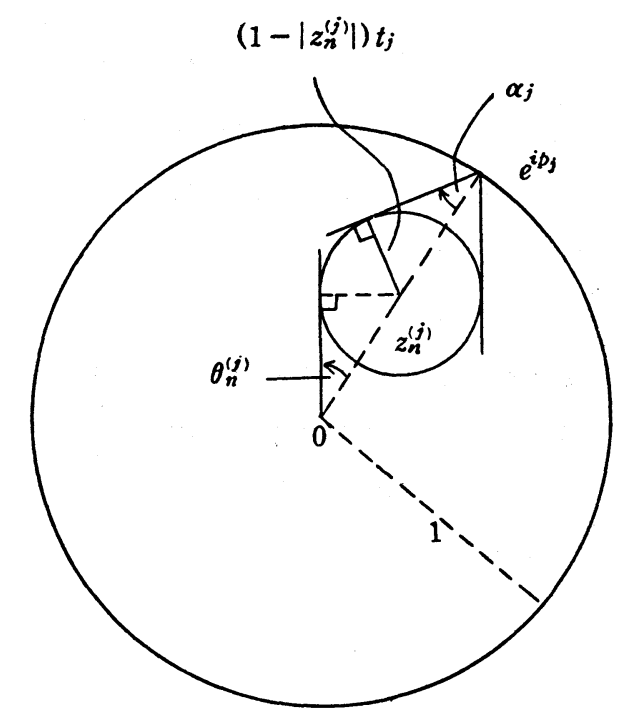

FIGURE 1

Since

$$
\sin \theta_{n}^{(j)}=\frac{\left(1-\left|z_{n}^{(j)}\right|\right) t_{j}}{\left|z_{n}^{(j)}\right|}=\frac{t_{j}}{n^{2}-1}
$$

and

$$
\theta_{n}^{(j)}<\frac{\pi}{2} \sin \theta_{n}^{(j)}
$$

then for $n \geq 2$, and all $j$,

$$
\theta_{n}^{(j)}<\frac{\pi}{2} \frac{t_{j}}{n^{2}-1}<\frac{\pi t_{j}}{n^{2}}
$$

Since the quadrilateral

$$
Q_{n}^{(j)}=\left\{r e^{i \theta} \mid p_{j}-\theta_{n}^{(j)} \leq \theta \leq p_{j}+\theta_{n}^{(j)}, 1-\left(\frac{1+t_{j}}{n^{2}}\right) \leq r \leq 1-\left[\frac{1-t_{j}}{n^{2}}\right]\right\}
$$


contains $D_{n}^{(j)}$ for each value $n$ and $j$, setting $z=r e^{i \theta}$

$$
\begin{aligned}
\iint_{n_{n}^{(j)}} U_{1}(z) r d r d \theta & =\iint_{D_{n}^{(j)}} \frac{1}{(1-r)^{1 / 3}} r d r d \theta \\
& \leq \iint_{Q_{n}^{(j)}} \frac{r}{(1-r)^{1 / 3}} d r d \theta \\
& <\frac{6 \theta_{n}^{(j)} n^{2 / 3}}{\left(1-t_{1}\right)^{1 / 3}} .
\end{aligned}
$$

By (6.3)

$$
\frac{\theta_{n}^{(j)} n^{2 / 3}}{\left(1-t_{1}\right)^{1 / 3}}<\frac{\pi^{t_{j}}}{n^{4 / 3}\left(1-t_{1}\right)^{1 / 3}}
$$

Thus (6.5) and (6.6) combine to give

$$
\begin{aligned}
\iint_{n} U_{1}(z) r d r d \theta & \leq \sum_{j=1}^{\infty} \sum_{n=1}^{\infty} \iint_{D_{n}^{(j)}} U_{1}(z) r d r d \theta \\
& <\sum_{j=1}^{\infty} \sum_{n=1}^{\infty} \frac{6 \pi t_{j}}{\left(1-t_{1}\right)^{1 / 3} n^{4 / 3}} \\
& =\frac{6 \pi}{\left(1-t_{1}\right)^{1 / 3}} \sum_{j=1}^{\infty} t_{j} \sum_{n=1}^{\infty} \frac{1}{n^{4 / 3}} .
\end{aligned}
$$

That this expression is finite follows from $(6.0)$; hence $U(z)$ is summable over $D$.

To conclude, we see that for any fixed value $j$, and any sequence $\left\{\zeta_{n}^{(j)}\right\}$, $\zeta_{n}^{(j)} \in D_{n}^{(j)}, n=1,2, \ldots, U_{1}\left(\zeta_{j}\right)\left(1-\left|\zeta_{j}\right|\right) \rightarrow \infty$ as $n \rightarrow \infty$. Thus the sets $M_{n}^{(t)}$ do not exist for the sequence $\left\{z_{n}^{(j)}\right\}$ and any $t \leq t_{j}$. Since this behavior is true for all values $j$ the exceptional set for $U_{1}(z)$ contains the set of points $e^{i p_{j}}, j=1$, $2, \ldots$.

Our next example concerns the behavior of a function $U_{2}(z)$ whose integral over $D$ diverges in some specified manner but for each $e^{i \theta} \in C$, there is a sequence tending to $e^{i \theta}$ and a value $t, 0<t<1$, for which the sets $M_{n}^{(t)}$ fail to exist.

For $0 \leq r<1$ let $\Psi(r)$ denote any real-valued function such that

I) $\Psi(r)$ is a non-decreasing function of $r, 0 \leq r<1$;

II) $\Psi(0)=0, \lim _{r \rightarrow 1} \Psi(r)=\infty$.

EXAMPLE 2. Let $\Psi(r)$ be an arbitrary function satisfying (6.7). Then there 
exists a real-valued, non-negative, measurable function $U_{2}(z)$ defined in $D$ with the property that the double integral of $U_{2}(z)$ over $D$ is infinite but, setting $z=r e^{i \theta}$,

$$
\int_{0}^{\rho} \int_{0}^{2 \pi} U_{2}(z) r d \theta d r<\Psi(\rho)
$$

for $0 \leq \rho<1$. Further for any $\theta \in[0,2 \pi]$ there is a sequence $\left\{z_{j}\right\}$ in $D$ which tends to $e^{i \theta}$ such that, if $\zeta_{j} \in D\left(z_{j},\left(1-\left|z_{j}\right|\right) 1 / 8\right)$, then

$$
\lim _{j \rightarrow \infty} U_{2}\left(\zeta_{j}\right)\left(1-\left|\zeta_{j}\right|\right)=\infty .
$$

Define a sequence of concentric, disjoint rings in $D$ as follows: let a sequence of positive numbers $\left\{n_{j}\right\}$ be chosen so that

$$
n_{j+1} \geq 2 n_{j},
$$

and

$$
2 \pi j^{2} \log 2 \leq \Psi\left(1-\frac{1}{n_{j}}\right), \quad j=1,2, \ldots
$$

That such a sequence exists follows from the monotonicity of $\Psi(r)$. Next let $R j$ be the ring $\left\{z \in D\left|1-\frac{1}{n_{j}} \leq\right| z \mid<1-\frac{1}{2 n_{j}}\right\} j=1,2, \ldots$ Note that these rings are disjoint by (6.8). Lastly select any function $\Psi^{*}(r)$ satisfying (6.7) and in addition

$$
1 \leq \Psi^{*}\left(1-\frac{1}{2 n_{j}}\right) \leq j \quad j=1,2, \ldots
$$

The desired function is

$$
U_{2}(z)=\left\{\begin{array}{l}
\frac{\Psi^{*}(|z|)}{1-|z|}, \quad z \in \bigcup_{j=1}^{\infty} R_{j} ; \\
0, z \in D-\bigcup_{j=1}^{\infty} R_{j} .
\end{array}\right.
$$

To demonstrate that the integral of $U_{2}(z)$ over $D$ diverges in the proper fashion fix a value $r_{0}, 0<r_{0}<1$, and let $j_{0}$ be chosen so that

$$
1-\frac{1}{n_{j_{0}}} \leq r_{0}<1-\frac{1}{n_{j_{0+1}}}
$$

Since $U_{2}(z)$ vanishes except on the ring $R_{j},(6.9),(6.10)$ and (6.11) imply

$$
\int_{0}^{r_{0}} \int_{0}^{2 \pi} U_{2}(z) r d \theta d r \leq \sum_{j=1}^{j 0} \iint_{R_{j}} \frac{\Psi^{*}(r) r d \theta d r}{1-|z|}
$$




$$
\begin{aligned}
& <2 \pi j_{0}^{2} \log 2 \\
& <\Psi\left(r_{0}\right)
\end{aligned}
$$

A short calculation yields that the integral of $U_{\mathbf{2}}(z)$ over $D$ is infinite.

Finally to exhibit sequences which have the desired properties consider, for any $0 \leq \theta<2 \pi$, the sequence $z_{j}=\left(1-\frac{3}{4} \frac{1}{n_{j}}\right) e^{i \theta}, j=1,2, \ldots$ The width of the ring $R_{j}$ is $\frac{1}{2 n_{j}}$. while the diameter of $D\left(z_{j},\left(1-\left|z_{j}\right|\right) 1 / 8\right)$ is $\frac{3}{16} \frac{1}{n_{j}}$; and since the point $z_{j}$ is equidistant from the boundary circles of $R_{j}$

$$
D\left(z_{j},\left(1-\left|z_{j}\right|\right) 1 / 8\right) \subset R_{j}, j=1,2, \ldots .
$$

From the definition of $U_{2}(z)$ and $\Psi^{*}(|z|)$ if $\zeta_{j} \in D\left(z_{j},\left(1-\left|z_{j}\right|\right) 1 / 8\right), j=1$, $2, \ldots$, then

$$
\lim _{j \rightarrow \infty} U_{2}\left(\zeta_{j}\right)\left(1-\left|\zeta_{j}\right|\right)=\infty
$$

The last example indicates that the rate of growth demonstrated in Theorem 1 cannot be improved.

ExAmple 3. Let $\Psi(r)$ be any function satisfying (6.7). Then there exists a real valued, nonnegative, measurable function $U_{3}(z)$ defined in $D$ for which

$$
\iint_{D} U_{3}(z) d x d y<\infty ;
$$

further for any $0 \leq \theta<2 \pi$ there is a sequence $\left\{z_{j}\right\}$ in $D$ tending radially to $e^{i \theta}$ with the property that if $\zeta_{j} \in D\left(z_{j},\left(1-\left|z_{j}\right|\right) 1 / 8\right), j=1,2, \ldots$, then

$$
\lim _{j \rightarrow \infty} U_{3}\left(\zeta_{j}\right)\left(1-\left|\zeta_{j}\right|\right) \Psi\left(\left|\zeta_{j}\right|\right)=\infty .
$$

Let $\Psi^{*}(r)$ be any function satisfying (6.7) and such that as $r \rightarrow 1$, $\Psi(r) / \Psi^{*}(r) \rightarrow \infty$. As before, define a sequence of concentric, disjoint rings $R_{j}$ in $D$ by first specifying a sequence of positive integers $\left\{n_{j}\right\}$ with

$$
\begin{aligned}
& \text { I) } \Psi^{*}\left(1-\frac{1}{n_{j}}\right) \geq j^{2} \text {; } \\
& \text { II) } n_{j+1} \geq 2 n_{j}
\end{aligned}
$$

for $j=1,2, \ldots$;

then set $R_{j}=\left\{z\left|1-\frac{1}{n_{j}} \leq\right| z \mid<1-\frac{1}{2 n_{j}}\right\}, j=1,2, \ldots$ Define 


$$
U_{3}(z)=\left\{\begin{array}{l}
\frac{1}{(1-|z|) \Psi^{*}(|z|)}, \quad z \in \bigcup_{j=1}^{\infty} R_{j} ; \\
0, z \in D-\bigcup_{j=1}^{\infty} R_{j} .
\end{array}\right.
$$

The finiteness of the double integral of $U_{3}(z)$ over $D$ follows from

$$
\begin{aligned}
\iint_{n} U_{3}(r) r d r d \theta & =\sum_{j=1} \iint_{R_{j}} \frac{r d r d \theta}{(1-r) \Psi^{*}(|z|)} \\
& <\sum_{j=1} \frac{2 \pi \log 2}{\Psi^{*}\left(1-\frac{1}{n_{j}}\right)} .
\end{aligned}
$$

By (6.12) the last term is less than or equal to

$$
\sum_{j=1}^{\infty} \frac{2 \pi \log 2}{j^{2}}
$$

For arbitrary $e^{i \theta}$ let $z_{j}=\left(1-\frac{3}{4} \frac{1}{n_{j}}\right) e^{i \theta}, j=1,2, \ldots$ As in the preceding example $D\left(z_{j},\left(1-\left|z_{j}\right|\right) 1 / 8\right) \subset R_{j}$ for all values $j$. Thus if $\zeta_{j} \in D\left(z_{j},\left(1-\left|z_{j}\right|\right) 1 / 8\right.$ )

$$
\lim _{j \rightarrow \infty} U_{3}\left(\zeta_{j}\right)\left(1-\left|\zeta_{j}\right|\right) \Psi^{*}\left(\left|\zeta_{j}\right|\right)=1
$$

then the definition of $\Psi^{*}(r)$ gives

$$
\lim _{j \rightarrow \infty} U_{\mathbf{3}}\left(\zeta_{j}\right)\left(1-\left|\zeta_{j}\right|\right) \Psi\left(\left|\zeta_{j}\right|\right)=\infty .
$$

This completes the proof of example 3 .

\section{REFERENCES}

[1] Gehring, F.. On the radial order of subharmonic functions, J. Math. Soc. Japan, Vol. 9 (1957), pp. 77-79.

[2] Lehto, O., and Virtanen, K., Boundary behavior and normal meromorphic functions, Acta Math. 97 (1957), pp. 47-65.

[3] Lelong-Ferrand, J., Representation conforme at transformations à integrale de Dirichlet bornee, Paris 1955.

[4] Rung, D. C., Results on the order of holomorphic functions defined in the unt disk, J. Math. Soc. Japan, Vol. 14, 3 (1962), pp. 322-332.

[5] Seidel, W., and Walsh, J. L., On the derivatives of functions analytic in the unit circle and their radii of univalence and of p-valence, Trans. Am. Math. Soc., Vol. 52 (1942), pp. 128-216.

Pennsylvania State University

University Park, Pennsylvania 